\title{
Front Matter: Volume 8679
}

, "Front Matter: Volume 8679," Proc. SPIE 8679, Extreme Ultraviolet (EUV) Lithography IV, 867901 (24 April 2013); doi: 10.1117/12.2027004

SPIE. Event: SPIE Advanced Lithography, 2013, San Jose, California, United States 


\section{PROCEEDINGS OF SPIE}

\section{Extreme Ultraviolet (EUV) Lithography IV}

Patrick P. Naulleau

Editor

25-28 February 2013

San Jose, California, United States

Sponsored by

SPIE

Cosponsored by

Cymer, Inc. (United States)

Published by

SPIE 
The papers included in this volume were part of the technical conference cited on the cover and title page. Papers were selected and subject to review by the editors and conference program committee. Some conference presentations may not be available for publication. The papers published in these proceedings reflect the work and thoughts of the authors and are published herein as submitted. The publisher is not responsible for the validity of the information or for any outcomes resulting from reliance thereon.

Please use the following format to cite material from this book:

Author(s), "Title of Paper," in Extreme Ultraviolet (EUV) Lithography IV, edited by Patrick P. Naulleau, Proceedings of SPIE Vol. 8679 (SPIE, Bellingham, WA, 2013) Article CID Number.

ISSN: 0277-786X

ISBN: 9780819494610

Published by

SPIE

P.O. Box 10, Bellingham, Washington 98227-0010 USA

Telephone +1 3606763290 (Pacific Time) · Fax +1 3606471445

SPIE.org

Copyright $@ 2013$, Society of Photo-Optical Instrumentation Engineers.

Copying of material in this book for internal or personal use, or for the internal or personal use of specific clients, beyond the fair use provisions granted by the U.S. Copyright Law is authorized by SPIE subject to payment of copying fees. The Transactional Reporting Service base fee for this volume is $\$ 18.00$ per article (or portion thereof), which should be paid directly to the Copyright Clearance Center (CCC), 222 Rosewood Drive, Danvers, MA 01923. Payment may also be made electronically through $\mathrm{CCC}$ Online at copyright.com. Other copying for republication, resale, advertising or promotion, or any form of systematic or multiple reproduction of any material in this book is prohibited except with permission in writing from the publisher. The CCC fee code is $0277-786 \mathrm{X} / 13 / \$ 18.00$.

Printed in the United States of America.

Publication of record for individual papers is online in the SPIE Digital Library.

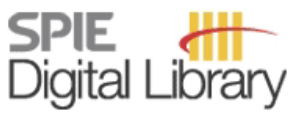

SPIEDigitalLibrary.org

Paper Numbering: Proceedings of SPIE follow an e-First publication model, with papers published first online and then in print and on CD-ROM. Papers are published as they are submitted and meet publication criteria. A unique, consistent, permanent citation identifier (CID) number is assigned to each article at the time of the first publication. Utilization of CIDs allows articles to be fully citable as soon as they are published online, and connects the same identifier to all online, print, and electronic versions of the publication. SPIE uses a six-digit CID article numbering system in which:

- The first four digits correspond to the SPIE volume number.

- The last two digits indicate publication order within the volume using a Base 36 numbering

system employing both numerals and letters. These two-number sets start with 00, 01, 02, 03, 04, $05,06,07,08,09,0 A, 0 B \ldots$. OZ, followed by 10-1Z, 20-2Z, etc.

The CID Number appears on each page of the manuscript. The complete citation is used on the first page, and an abbreviated version on subsequent pages. Numbers in the index correspond to the last two digits of the six-digit CID Number. 


\title{
Contents
}

\section{Part One}

\author{
xv Conference Committee
}

SESSION 1 INVITED I

867903 Effects of multilayer period on EUVL imaging for 2X node and beyond (Invited Paper) [8679-2]

S.-Y. Lee, H.-S. Seo, T.-G. Kim, S.-H. Kim, R. Chalykh, S.-S. Kim, C.-U. Jeon, SAMSUNG

Electronics Co., Ltd. (Korea, Republic of)

867904 Investigation of EUV pellicle feasibility (Invited Paper) [8679-3]

L. Scaccabarozzi, D. Smith, P. R. Diago, E. Casimiri, N. Dziomkina, H. Meijer, ASML

Netherlands B.V. (Netherlands)

867905 EUV resist materials design for 15nm half pitch and below (Invited Paper) [8679-4]

H. Tsubaki, S. Tarutani, N. Inoue, H. Takizawa, T. Goto, FUJIFILM Corp. (Japan)

\section{SESSION 2 EUV RESISTS: JOINT SESSION WITH CONFERENCES 8679 AND 8682}

867906 Oxide nanoparticle EUV resists: toward understanding the mechanism of positive and negative tone patterning [8679-5]

S. Chakrabarty, C. Ouyang, M. Krysak, M. Trikeriotis, Cornell Univ. (United States); K. Cho,

SEMATECH Inc. (United States); E. P. Giannelis, C. K. Ober, Cornell Univ. (United States)

$867907 \quad$ Effect of leaving group design on EUV lithography performance [8679-6]

O. Ongayi, V. Jain, S. Coley, D. Valeri, A. Kwok, D. Quach, M. Wagner, J. Cameron,

J. Thackeray, Dow Electronic Materials (United States)

\section{SESSION 3 SOURCES}

8679 OA LPP-EUV light source development for high volume manufacturing lithography [8679-9]

H. Mizoguchi, H. Nakarai, T. Abe, T. Ohta, K. M. Nowak, Y. Kawasuji, H. Tanaka,

Y. Watanabe, T. Hori, T. Kodama, Y. Shiraishi, T. Yanagida, T. Yamada, T. Yamazaki,

S. Okazaki, T. Saitou, Gigaphoton Inc. (Japan)

8679 OB Advances in computer simulations of LPP sources for EUV lithography [8679-10]

A. Hassanein, T. Sizyuk, Purdue Univ. (United States) 
8679 OC Lifetime and refurbishment of multilayer LPP collector mirrors [8679-12]

T. Feigl, M. Perske, H. Pauer, T. Fiedler, S. Yulin, N. Kaiser, A. Tünnermann, Fraunhofer-Institut für Angewandte Optik und Feinmechanik (Germany); N. R. Böwering, A. I. Ershov, S. de Dea, K. Hoffmann, B. La Fontaine, I. V. Fomenkov, D. C. Brandt, Cymer, Inc. (United States)

8679 OD Contamination concerns at the intermediate focus of an extreme ultraviolet light source [8679-13]

D. N. Ruzic, J. Sporre, D. Elg, D. Curreli, Univ. of Illinois at Urbana-Champaign (United States)

\section{SESSION 4 MASK I}

8679 OF Dressed-photon nanopolishing for extreme ultraviolet mask substrate defect mitigation [8679-14]

R. Teki, A. J. Kadaksham, F. Goodwin, SEMATECH Inc. (United States); T. Yatsui, The Univ. of Tokyo (Japan) and Japan Science and Technology Agency (Japan); M. Ohtsu, The Univ. of Tokyo (Japan)

8679 OG EUV mask defect analysis from mask to wafer printing [8679-15] Y. Hyun, K. Seo, K. Kim, I. Lee, B. Lee, S. Koo, J. Lee, S. Kim, S. Kim, M. Kim, H. Kang, Hynix Semiconductor Inc. (Korea, Republic of)

$8679 \mathrm{OH}$ Defect printability comparing actinic printing with advanced simulation for EUV masks [8679-16]

I.-Y. Jang, R. Teki, V. Jindal, F. Goodwin, SEMATECH Inc. (United States); M. Satake, Y. Li, D. Peng, Luminescent Technologies (United States); S. Huh, S.-S. Kim, SAMSUNG Electronics Co., Ltd. (Korea, Republic of)

8679 Ol EUV actinic blank inspection: from prototype to production [8679-17]

A. Tchikoulaeva, Lasertec U.S.A., Inc. Zweigniederlassung Deutschland (Germany);

H. Miyai, T. Suzuki, K. Takehisa, H. Kusunose, Lasertec Corp. (Japan); T. Yamane, T. Terasawa, H. Watanabe, S. Inove, I. Mori, EUVL Infrastructure Development Ctr., Inc. (Japan)

\section{SESSION 5 RESIST OUTGASSING}

8679 OK Relationship between resist related outgassing and witness sample contamination in the NXE outgas qualification using electrons and EUV [8679-19]

I. Pollentier, R. Lokasani, R. Gronheid, IMEC (Belgium); S. Hill, C. Tarrio, T. Lucatorto, National Institute of Standards and Technology (United States)

$8679 \mathrm{OL}$ Resist outgassing contamination growth results using both photon and electron exposures [8679-20]

G. Denbeaux, Y. Kandel, G. Kane, D. Alvardo, M. Upadhyaya, Y. Khopkar, Univ. at Albany (United States); A. Friz, K. Petrillo, J. Sohn, C. Sarma, D. Ashworth, SEMATECH Inc. (United States) 
8679 OM Study of EUV outgassing spatial distribution toward witness plate in the EUV outgas tester [8679-21]

Y. Kikuchi, E. Shiobara, H. Tanaka, I. Takagi, K. Katayama, N. Sugie, T. Takahashi, S. Inoue, EUVL Infrastructure Development Ctr., Inc. (Japan); T. Watanabe, T. Harada, H. Kinoshita, Univ. of Hyogo (Japan)

867900 Balancing lithographic performance and resist outgassing in EUV resists [8679-23]

S.-H. Chang, S.-F. Chen, Y.-Y. Chen, M.-C. Chien, S.-C. Chien, T.-L. Lee, J. J.-H. Chen, A. Yen, TSMC Inc. (Taiwan)

\section{SESSION 6 OPTICS AND METROLOGY}

8679 OP EUVL resist-based aberration metrology [8679-24]

G. L. Fenger, Rochester Institute of Technology (United States); S. Raghunathan, L. Sun, O. R. Wood, GLOBALFOUNDRIES Inc. (United States); B. W. Smith, Rochester Institute of Technology (United States)

$86790 Q \quad$ In-situ optical testing of exposure tools via localized wavefront curvature sensing [8679-25] R. Miyakawa, Lawrence Berkeley National Lab. (United States); X. Zhou, SEMATECH Inc. (United States); M. Goldstein, SEMATECH Inc. (United States) and Intel Corp. (United States): D. Ashworth, K. Cummings, Y.-J. Fan, SEMATECH Inc. (United States); Y. Shroff, Intel Corp. (United States); G. Denbeaux, Y. Kandel, Univ. at Albany (United States); P. Naulleau, Lawrence Berkeley National Lab. (United States)

8679 OT Application of phase shift focus monitor in EUVL process control [8679-28]

L. Sun, S. Raghunathan, GLOBALFOUNDRIES Inc. (United States); V. Jindal, SEMATECH Inc. (United States); E. Gullikson, Lawrence Berkeley National Lab. (United States); P. Mangat, GLOBALFOUNDRIES Inc. (United States); I. Mochi, K. A. Goldberg, M. P. Benk, Lawrence Berkeley National Lab. (United States); O. Kritsun, T. Wallow, D. Civay, O. Wood, GLOBALFOUNDRIES Inc. (United States)

\section{SESSION 7 OPC AND MODELING}

8679 OU EUV multilayer defect compensation (MDC) by absorber pattern modification, film deposition, and multilayer peeling techniques [8679-29]

L. Pang, M. Satake, Y. Li, P. Hu, D. Peng, D. Chen, V. Tolani, Luminescent Technologies (United States)

8679 OV Evaluation of methods to improve EUV OPC model accuracy [8679-30]

T. H. Coskun, C. Clifford, G. Fenger, GLOBALFOUNDRIES Inc. (United States); G. S. Chua, GLOBALFOUNDRIES Singapore (Singapore); K. Standiford, R. Schlief, C. Higgins, Y. Zou, GLOBALFOUNDRIES Inc. (United States)

8679 OW Fast 3D thick mask model for full-chip EUVL simulations [8679-31]

P. Liu, X. Xie, W. Liu, K. Gronlund, ASML Brion Technologies (United States) 
$86790 \mathrm{X}$ Process and proximity correction, and verification for extreme ultraviolet lithography [8679-32]

G. Perçin, Cadence Design Systems, Inc. (United States); H. Dai, Applied Materials, Inc. (United States); H.-T. Huang, A. Liu, A. Mokhberi, X. Zheng, Cadence Design Systems, Inc. (United States); C. Ngai, Applied Materials, Inc. (United States)

8679 OY Modeling strategies for EUV mask multilayer defect dispositioning and repair [8679-33] A. Erdmann, P. Evanschitzky, Fraunhofer Institute for Integrated Systems and Device Technology (Germany); T. Bret, Carl Zeiss SMS GmbH (Germany); R. Jonckheere, IMEC (Belgium)

\section{SESSION 8 EUV RESISTS}

867910 Evaluation of EUV resist performance with interference lithography towards $11 \mathrm{~nm}$ halfpitch and beyond [8679-35]

Y. Ekinci, M. Vockenhuber, M. Hojeij, L. Wang, N. Mojarad, Paul Scherrer Institute (Switzerland)

867911 Resist process applications to improve EUV patterning [8679-36] K. Petrillo, K. Cho, A. Friz, C. Montgomery, D. Ashworth, M. Neisser, S. Wurm, SEMATECH Inc. (United States); T. Saito, L. Huli, A. Ko, A. Metz, TEL Technology Ctr., America, LLC (United States)

867912 Development of molecular resist derivatives for EUV lithography [8679-37]

D. P. Green, The Dow Chemical Co. (United States); V. Jain, Dow Electronic Materials (United States); B. Bailey, The Dow Chemical Co. (United States); M. Wagner, Dow Electronic Materials (United States); M. Clark, Dow Advanced Materials (United States); D. Valeri, Dow Electronic Materials (United States); S. Lakso, The Dow Chemical Co. (United States)

867913 Relationship between stochastic effect and resist pattern defect in extreme ultraviolet lithography [8679-38]

T. Kozawa, Osaka Univ. (Japan); J. J. Santillan, T. Itani, EUVL Infrastructure Development Ctr., Inc. (Japan)

867914 EUV sensitive Si containing hard mask (Si-HM) for PTD and NTD process in EUVL [8679-39] W. Shibayama, S. Shigaki, R. Sakamoto, R. Onishi, H. Yaguchi, Nissan Chemical Industries, Ltd. (Japan); B. C. Ho, Nissan Chemical Taiwan (Taiwan)

867915 Mask effects for high-NA EUV: impact of NA, chief-ray-angle, and reduction ratio (Invited Paper) [8679-40]

J. T. Neumann, P. Gräupner, W. Kaiser, R. Garreis, Carl Zeiss SMT GmbH (Germany); B. Geh, Carl Zeiss SMT (United States) 
867916 Considerations for high-numerical aperture EUV lithography (Invited Paper) [8679-41]

H. J. Levinson, P. Mangat, T. Wallow, L. Sun, P. Ackmann, S. Meyers, GLOBALFOUNDRIES Inc. (United States)

867917 Projection optics for extreme ultraviolet lithography (EUVL) micro-field exposure tools (METs) with a numerical aperture of 0.5 [8679-42]

H. Glatzel, Zygo Corp. (United States); D. Ashworth, SEMATECH Inc. (United States); M. Bremer, R. Chin, Zygo Corp. (United States); K. Cummings, SEMATECH Inc. (United States); L. Girard, Zygo Corp. (United States); M. Goldstein, SEMATECH Inc. (United States); E. Gullikson, Lawrence Berkeley National Lab. (United States); R. Hudyma, Hyperion Development LLC (United States); J. Kennon, B. Kestner, L. Marchetti, Zygo Corp. (United States); P. Naulleau, Lawrence Berkeley National Lab. (United States); R. Soufli, Lawrence Livermore National Lab. (United States); E. Spiller, Spiller X-Ray Optics (United States)

867918 Mask 3D effects and compensation for high NA EUV lithography [8679-43] S. Raghunathan, GLOBALFOUNDRIES Inc. (United States); G. McIntyre, IBM Corp. (United States); G. Fenger, O. Wood, GLOBALFOUNDRIES Inc. (United States)

\section{SESSION $10 \quad$ MASK II}

867919 Commissioning an EUV mask microscope for lithography generations reaching $8 \mathrm{~nm}$ [8679-44]

K. A. Goldberg, I. Mochi, M. Benk, A. P. Allezy, M. R. Dickinson, C. W. Cork, D. Zehm, J. B. Macdougall, E. Anderson, F. Salmassi, W. L. Chao, V. K. Vytla, E. M. Gullikson, J. C. DePonte, M. S. G. Jones, D. Van Camp, J. F. Gamsby, W. B. Ghiorso, H. Huang, W. Cork, E. Martin, Lawrence Berkeley National Lab. (United States); E. Van Every, E. Acome, Advanced Design Consulting USA, Inc. (United States); V. Milanovic, Mirrorcle Technologies, Inc. (United States); R. Delano, P. P. Naulleau, S. B. Rekawa, Lawrence Berkeley National Lab. (United States)

8679 1B Experimental phase defect printability evaluation using a programmed phase defect in EUVL mask [8679-46]

T. Terasawa, T. Amano, S. Oh, T. Yamane, H. Watanabe, EUVL Infrastructure Development Ctr., Inc. (Japan)

8679 ID Challenges in EUV mask blank deposition for high volume manufacturing [8679-48] V. Jindal, P. Kearney, A. Antohe, M. Godwin, A. John, R. Teki, J. Harris-Jones, E. Stinzianni, F. Goodwin, SEMATECH Inc. (United States)

8679 IE Extending Ru capping layer durability under physical force cleaning [8679-49] S. Singh, U. Dietze, SUSS MicroTec Inc. (United States); P. Dress, SUSS MicroTec Photomask Equipment GmbH \& Co. KG (Germany) 


\section{Part Two}

SESSION 11 INVITED II

8679 IF ASML's NXE plafform performance and volume introduction (Invited Paper) [8679-50] R. Peeters, S. Lok, E. van Alphen, N. Harned, ASML Netherlands B.V. (Netherlands); P. Kuerz, M. Lowisch, Carl Zeiss SMT AG (Germany); H. Meijer, D. Ockwell, E. van Setten, G. Schiffelers, J.-W. van der Horst, J. Stoeldraijer, R. Kazinczi, R. Droste, H. Meiling, R. Kool, ASML Netherlands B.V. (Netherlands)

$86791 \mathrm{G} \quad \mathrm{CO}_{2} /$ Sn LPP EUV sources for device development and HVM (Invited Paper) [8679-51]

D. C. Brandt, I. V. Fomenkov, N. R. Farrar, B. La Fontaine, D. W. Myers, D. J. Brown, A. I. Ershov, R. L. Sandstrom, G. O. Vaschenko, N. R. Böwering, P. Das, V. B. Fleurov, K. Zhang, S. N. Srivastava, I. Ahmad, C. Rajyaguru, S. De Dea, W. J. Dunstan, P. Baumgart, T. Ishihara, R. D. Simmons, R. N. Jacques, R. A. Bergstedt, P. I. Porshnev, C. J. Wittak, R. J. Rafac, J. Grava, A. A. Schafgans, Y. Tao, K. Hoffmann, T. Ishikawa, D. R. Evans, S. D. Rich, Cymer, Inc. (United States)

8679 1H Optics for ASML's NXE:3300B platform (Invited Paper) [8679-52] M. Lowisch, P. Kuerz, O. Conradi, G. Wittich, W. Seitz, W. Kaiser, Carl Zeiss SMT GmbH (Germany)

867911 Through-focus EUV multilayer defect repair with nanomachining (Invited Paper) [8679-53] G. McIntyre, E. Gallagher, IBM Microelectronics (United States); T. Robinson, RAVE LLC (United States); A. C. Smith, M. Lawliss, IBM Microelectronics (United States); J. LeClaire, R. Bozak, R. White, M. Archuletta, RAVE LLC (United States)

$86791 \mathrm{~J}$ Patterning challenges of EUV lithography for 1X-nm node DRAM and beyond (Invited Paper) [8679-54] T.-S. Eom, H.-I. Kim, C.-K. Kang, Y.-J. Ryu, S.-H. Hwang, H.-H. Lee, H.-Y. Lim, J.-S. Park, N.-J. Kwak, S. Park, Hynix Semiconductor Inc. (Korea, Republic of)

$86791 \mathrm{~K}$ Towards manufacturing a 10nm node device with complementary EUV lithography (Invited Paper) [8679-55]

J. V. Hermans, IMEC (Belgium); H. Dai, Applied Materials, Inc. (United States);

A. Niroomand, Micron Technology, Inc. (United States); D. Laidler, M. Mao, IMEC (Belgium); Y. Chen, Applied Materials, Inc. (United States); P. Leray, IMEC (Belgium); C. Ngai, Applied Materials, Inc. (United States); S. Cheng, IMEC (Belgium)

\section{POSTER SESSION}

$86791 \mathrm{~L} \quad$ Limitation of OAI + AttPSM in EUVL [8679-56]

S.-S. YU, Y.-C. LU, C.-T. Shih, J. J. H. Chen, A. Yen, Taiwan Semiconductor Manufacturing Co. Ltd. (Taiwan) 
8679 1M Experimental verification of EUV mask limitations at high numerical apertures [8679-57] R. Chao, Lawrence Berkeley National Lab. (United States) and Univ. of California, Berkeley (United States); P. Graeupner, Carl Zeiss SMT GmbH (Germany); E. Gullikson, Lawrence Berkeley National Lab. (United States); S.-S. Kim, SAMSUNG Electronics Co., Ltd. (Korea, Republic of); J.-T. Neumann, Carl Zeiss SMT GmbH (Germany); R. Miyakawa, Lawrence Berkeley National Lab. (United States); H.-S. Seo, SAMSUNG Electronics Co., Ltd. (Korea, Republic of); A. Neureuther, Univ. of California, Berkeley (United States); P. Naulleau, Lawrence Berkeley National Lab. (United States)

$86791 \mathrm{~N}$ Homogeneity improvement of $\mathrm{TiO}_{2}-\mathrm{SiO}_{2}$ glass synthesized by the soot method and its evaluation using the ultrasonic measurement system [8679-58]

M. Kawagishi, J. Konishi, M. Takata, Asahi Glass Co., Ltd. (Japan); M. Arakawa, Y. Ohashi, J. Kushibiki, Tohoku Univ. (Japan)

867910 Inspection and compositional analysis of sub-20 nm EUV mask blank defects by thin film decoration technique [8679-59]

V. Jindal, A. John, J. Harris-Jones, P. Kearney, A. Antohe, E. Stinzianni, F. Goodwin,

SEMATECH Inc. (United States); T. Onove, HOYA Corp. (Japan)

8679 1P Propagation of surface topography of EUV blank substrate through multilayer and impact of phase defect structure on wafer image [8679-60]

T. Amano, T. Terasawa, EUVL Infrastructure Development Ctr., Inc. (Japan)

8679 1Q Modeling studies on alternative EUV mask concepts for higher NA [8679-61]

A. Erdmann, T. Fühner, P. Evanschitzky, Fraunhofer Institute for Integrated Systems and

Device Technology (Germany); J. T. Neumann, J. Ruoff, P. Gräupner, Carl Zeiss SMT GmbH (Germany)

8679 IR Low thermal expansion material (LTEM) cleaning and optimization for extreme ultraviolet (EUV) blank deposition [8679-62]

A. J. Kadaksham, R. Teki, M. Godwin, M. House, F. Goodwin, SEMATECH Inc. (United States)

8679 IT Evaluation of novel projection electron microscopy (PEM) optics for EUV mask inspection [8679-64]

R. Hirano, S. lida, T. Amano, T. Terasawa, H. Watanabe, EUVL Infrastructure Development Ctr., Inc. (Japan); K. Terao, EBARA Corp. (Japan)

$86791 \mathrm{U}$ Registration accuracy improvement of fiducial mark on EUVL mask with MIRAI EUV ABI prototype [8679-65]

T. Murachi, T. Amano, EUVL Infrastructure Development Ctr., Inc. (Japan)

$86791 \mathrm{~V}$ Study on EUV mask defect inspection with hp 16nm node using simulated projection electron microscope images [8679-66]

S. lida, T. Amano, R. Hirano, T. Terasawa, H. Watanabe, EUVL Infrastructure Development Ctr., Inc. (Japan)

8679 IW Development of 3D Monte Carlo simulations for predicting multilayer geometry of pit-type EUV defects [8679-67]

R. F. Spivey, Rensselaer Polytechnic Institute (United States); R. Teki, SEMATECH Inc. (United States); T.-M. Lu, Rensselaer Polytechnic Institute (United States) 
8679 1X Effect of phase defect shape on $A B I$ signal intensity and defect image intensity on wafer with simulation [8679-68]

N. Takagi, T. Terasawa, Y. Arisawa, EUVL Infrastructure Development Ctr., Inc. (Japan)

8679 1Y Mathematical model for calculating speckle contrast through focus [8679-69]

R. A. Claus, A. R. Neureuther, Univ. of California, Berkeley (United States); P. P. Naulleau, Lawrence Berkeley National Lab. (United States)

867912 Evaluating the effect of EUV multilayer buried defects on feature printability using a stochastic resist model [8679-70]

T. Graves, J. Biafore, M. Smith, S. Robertson, C. Fang, KLA-Tencor Corp. (United States)

867920 Effects of varying the parameters in witness-sample-based photoresist outgas testing: dependence of the carbon growth on pumping speed and the dose, time, and area of resist exposure [8679-71]

C. Tarrio, R. F. Berg, S. B. Hill, S. Grantham, N. S. Faradzhev, T. B. Lucatorto, National Institute of Standards and Technology (United States)

867921 Status of EUV reflectometry at PTB [8679-72]

C. Laubis, A. Barboutis, M. Biel, C. Buchholz, B. Dubrau, A. Fischer, A. Hesse, J. Puls,

C. Stadelhoff, V. Soltwisch, F. Scholze, Physikalisch-Technische Bundesanstalt (Germany)

867922 Hartmann wavefront sensor for EUV radiation [8679-73]

K. Mann, B. Flöter, T. Mey, B. Schäfer, Laser-Lab. Göttingen e.V. (Germany); B. Keitel,

E. Plönjes, K. Tiedtke, Deutsches Elektronen-Synchrotron (Germany)

867923 A reverse design method for EUV lithography illumination system [8679-74]

Q. Mei, Y. Li, F. Liu, Beijing Institute of Technology (China)

867924 Patterning at $6.5 \mathrm{~nm}$ wavelength using interference lithography [8679-75]

N. Mojarad, M. Vockenhuber, L. Wang, B. Terhalle, Y. Ekinci, Paul Scherrer Institute (Switzerland)

867925 Simulation analysis of LER and dose tradeoffs for EUV resists with photo-decomposable quenchers [8679-76]

S. Bhattarai, A. R. Neureuther, Univ. of California, Berkeley (United States) and Lawrence Berkeley National Lab. (United States); P. P. Naulleau, Lawrence Berkeley National Lab. (United States)

867926 Relation between sensitivity and resolution in polymer bound PAGs and polymer blend PAGs [8679-77]

S. Enomoto, T. N. Dang, C. Q. Dinh, S. Tagawa, Osaka Univ. (Japan) and Japan Science and Technology Agency (Japan)

867928 Stochastic resist patterning simulation using attenuated PSM for EUV lithography [8679-79] S. Hong, S. Jeong, J. U. Lee, S. M. Lee, J. Kim, Hanyang Univ. (Korea, Republic of); J. Doh, SAMSUNG Electronics Co., Ltd. (Korea, Republic of); J. Ahn, Hanyang Univ. (Korea, Republic of) 
867929 Quencher distribution engineering for out-of-band insensitive EUV resists: experiments and stochastic simulation [8679-80]

S.-C. Chien, S.-H. Chang, J.-C. Wu, J. J.-H. Chen, A. Yen, Taiwan Semiconductor

Manufacturing Co. Ltd. (Taiwan)

8679 2A Prediction of resist sensitivity for 13.5-nm EUV and 6.X-nm EUV extension from sensitivity for EBL [8679-81]

T. G. Oyama, Japan Atomic Energy Agency (Japan); A. Oshima, T. N. Dang, Osaka Univ. (Japan) and JST-CREST (Japan); S. Enomoto, Osaka Univ. (Japan); M. Washio, Waseda Univ. (Japan); S. Tagawa, Osaka Univ. (Japan) and JST-CREST (Japan)

8679 2B Study of LWR reduction and pattern collapse suppression for $16 \mathrm{~nm}$ node EUV resists [8679-82]

E. Shiobara, Y. Kikuchi, T. Itani, EUVL Infrastructure Development Ctr., Inc. (Japan)

8679 2C Reaction mechanisms of various chemically amplified EUV and EB resist [8679-83]

S. Enomoto, A. Oshima, S. Tagawa, Osaka Univ. (Japan) and JST-CREST (Japan)

8679 2D Development of an atomic hydrogen system for treatment of EUV mask blanks [8679-84]

T. R. Mowll, Univ. at Albany (United States); A. J. Kadaksham, SEMATECH Inc. (United

States); Z. R. Robinson, Univ. at Albany (United States) and SEMATECH Inc. (United States);

S. Mead, C. A. Ventrice Jr., Univ. at Albany (United States); F. Goodwin, SEMATECH Inc.

(United States)

$86792 \mathrm{E} \quad$ Resist outgassing characterization based on the resist compositions and process [8679-85] N. Sugie, T. Takahashi, K. Katayama, I. Takagi, Y. Kikuchi, H. Tanaka, E. Shiobara, S. Inove, EUVL Infrastructure Development Ctr., Inc. (Japan)

$86792 \mathrm{H} \quad$ Collector optic in-situ Sn removal using hydrogen plasma [8679-88]

J. R. Sporre, D. Elg, D. N. Ruzic, Univ. of Illinois at Urbana-Champaign (United States);

S. N. Srivastava, I. V. Fomenkov, D. C. Brandt, Cymer, Inc. (United States)

867921 Laser produced plasma EUV light source for EUVL patterning at $20 \mathrm{~nm}$ node and beyond [8679-89]

I. V. Fomenkov, D. C. Brandt, N. R. Farrar, B. La Fontaine, N. R. Böwering, D. J. Brown, A. I. Ershov, D. W. Myers, Cymer, Inc. (United States)

8679 2K Important processes in modeling and optimization of EUV lithography sources [8679-91] T. Sizyuk, A. Hassanein, Purdue Univ. (United States)

8679 2M Magnetic mitigation of debris for EUV sources [8679-93]

D. Elg, J. Sporre, D. Curreli, D. N. Ruzic, Univ. of Illinois at Urbana-Champaign (United

States); K. R. Umstadter, KLA-Tencor Corp. (United States) 
$86792 \mathrm{~N} \quad$ Enhancing resolution with pupil filtering for projection printing systems with fixed or restricted illumination angular distribution [8679-94]

G. McIntyre, IBM Corp. (United States); L. Teeuwen, ASML US, Inc. (United States); E. Sohmen, Carl Zeiss SMT GmbH (Germany); O. Wood, GLOBALFOUNDRIES Inc. (United States); D. Corliss, IBM Corp. (United States); T. van den Akker, S. Bouten, E. van Setten, O. Voznyi, S.-I. Han, ASML US, Inc. (United States); H. Bieg, Carl Zeiss SMT GmbH (Germany); M. Burkhardt, K. Petrillo, Z. J. Qi, A. Friz, IBM Corp. (United States)

867920 Roughness and variability in EUV lithography: Who is to blame? (part 1) [8679-95] A. Vaglio Pret, R. Gronheid, IMEC (Belgium); T. R. Younkin, IMEC (Belgium) and Intel Corp. (United States); G. Winroth, IMEC (Belgium); J. J. Biafore, KLA-Tencor Corp. (United States); Y. Anno, K. Hoshiko, IMEC (Belgium) and JSR Micro N.V. (Belgium); V. Constantoudis, National Ctr. for Scientific Research Demokritos (Greece)

$86792 \mathrm{~T} \quad 7 \mathrm{~nm}$ node EUV predictive study of mask LER transference to wafer [8679-97]

D. Civay, GLOBALFOUNDRIES Inc. (United States); E. Nash, U. Klostermann, Synopsys GmbH (Germany); T. Wallow, P. Mangat, H. P. Koh, GLOBALFOUNDRIES Inc. (United States); P. Brooker, Synopsys (United States); J. Siebert, Synopsys GmbH (Germany); H. J. Levinson, GLOBALFOUNDRIES Inc. (United States)

$86792 R \quad$ Silica aerogel can capture flying particles in EUV tools [8679-98]

K. Ota, J. Inove, Nikon Corp. (Japan)

8679 2S Impact of EUV mask roughness on lithography performance [8679-99]

Y. Arisawa, T. Terasawa, H. Watanabe, EUVL Infrastructure Development Ctr., Inc. (Japan)

8679 2T Track processing optimizations for different EUV resist platforms: preparing for a NXE:3300 baseline process [8679-100]

P. Foubert, IMEC (Belgium); K. Matsunaga, IMEC (Belgium) and Tokyo Electron Kyushu Ltd. (Japan); H. Shite, T. Shimoaoki, Tokyo Electron Kyushu Ltd. (Japan); K. Nafus, IMEC (Belgium) and Tokyo Electron Kyushu Ltd. (Japan); A.-M. Goethals, D. Van Den Heuvel, J. Hermans, E. Hendrickx, IMEC (Belgium); H. Kosugi, Tokyo Electron Kyushu Ltd. (Japan)

$86792 \mathrm{U}$ Simulation-assisted layout biasing in EUV lithography and prediction of an optimum resist parameter space [8679-101]

C. Sarma, SEMATECH Inc. (United States); J. Biafore, KLA-Tencor Corp. (United States);

K. Cho, K. Petrillo, M. Neisser, SEMATECH Inc. (United States)

8679 2V Deep ultraviolet out-of-band characterization of EUVL scanners and resists [8679-102] G. F. Lorusso, IMEC (Belgium); T. Matsumiya, J. Iwashita, T. Hirayama, Tokyo Ohka Kogyo Co., Ltd. (Japan); E. Hendrickx, IMEC (Belgium)

$86792 \mathrm{~W}$ Investigation of coat-develop track system for EUV resist processing [8679-104] M. Harumoto, O. Tamada, T. Miyagi, K. Kaneyama, A. Morita, C. Pieczulewski, M. Asai, SOKUDO Co. Ltd. (Japan)

$86792 X \quad$ Heat behavior of extreme-ultraviolet pellicle including mesh support [8679-105]

I.-S. Kim, E.-J. Kim, J.-W. Kim, H.-K. Oh, Hanyang Univ. (Korea, Republic of) 
$86792 Y$ The need for EUV lithography at advanced technology for sustainable wafer cost [8679-107]

A. Mallik, W. Vansumere, J. Ryckaert, A. Mercha, N. Horiguchi, S. Demuynck, J. Bömmels, T. Zsolt, G. Vandenberghe, K. Ronse, A. Thean, D. Verkest, H. Lebon, A. Steegen, IMEC (Belgium)

$86792 Z$ The study of novel PAG containing acid amplifier in EUV resist material [8679-108] H. S. Joo, J. H. Kim, J. H. Han, C. W. Bae, J. B. Shin, H. S. Lim, S. D. Cho, S. M. Kim, Korea Kumho Petrochemical Co., Ltd. (Korea, Republic of)

867934 Optimizing XPS tool performance for characterizing trace contamination elements for EUV resist outgas testing [8679-113]

M. Upadhyaya, Y. Kandel, G. Denbeaux, Univ. at Albany (United States); C. Montgomery, Y.-J. Fan, SEMATECH Inc. (United States)

867937 Effect of cleaning on EUV masks [8679-116]

J. W. Roberts, n\&k Technology, Inc. (United States)

Author Index 
Proc. of SPIE Vol. $8679867901-14$

Downloaded From: https://www.spiedigitallibrary.org/conference-proceedings-of-spie on 26 Apr 2023 Terms of Use: https://www.spiedigitallibrary.org/terms-of-use 


\section{Conference Committee}

Symposium Chair

Harry J. Levinson, GLOBALFOUNDRIES Inc. (United States)

Symposium Cochair

Mircea V. Dusa, ASML US, Inc. (United States)

Conference Chair

Patrick P. Naulleau, Lawrence Berkeley National Laboratory (United States)

Conference Cochair

Obert R. Wood II, GLOBALFOUNDRIES Inc. (United States)

Conference Program Committee

Markus Bender, Advanced Mask Technology Center GmbH Company KG (Germany)

Jos P. Benschop, ASML Netherlands B.V. (Netherlands)

Robert L. Brainard, University at Albany (United States)

Li-Jui Chen, Taiwan Semiconductor Manufacturing Company Ltd. (Taiwan)

Daniel A. Corliss, IBM Corporation (United States)

Emily E. Gallagher, IBM Corporation (United States)

Michael Goldstein, SEMATECH Inc. (United States)

Frank Goodwin, SEMATECH Inc. (United States)

Naoya Hayashi, Dai Nippon Printing Company, Ltd. (Japan)

Soichi Inove, EUVL Infrastructure Development Center, Inc. (Japan)

Bryan S. Kasprowicz, Photronics, Inc. (United States)

Seong-Sue Kim, SAMSUNG Electronics Company, Ltd.

(Korea, Republic of)

Bruno La Fontaine, Cymer, Inc. (United States)

Michael J. Lercel, SEMATECH Inc. (United States)

Ted Liang, Intel Corporation (United States)

Akira Miyake, Canon Inc. (Japan)

Hiroaki Morimoto, Toppan Printing Company, Ltd. (Japan)

Katsuhiko Murakami, Nikon Corporation (Japan)

Chris Ngai, Applied Materials, Inc. (United States)

Shinji Okazaki, Gigaphoton Inc. (Japan)

Uzodinma Okoroanyanwu, GLOBALFOUNDRIES Inc. (Germany) 
Eric M. Panning, Intel Corporation (United States)

Jan Hendrik Peters, Carl Zeiss SMS GmbH (Germany)

Jorge J. Rocca, Colorado State University (United States)

Kurt G. Ronse, IMEC (Belgium)

Tsutomu Shoki, HOYA Corporation (Japan)

Stanley E. Stokowski, KLA-Tencor Corporation (United States)

Anna V. Tchikoulaeva, Lasertec U.S.A., Inc. Zweigniederlassung

Deutschland (Germany)

Thomas I. Wallow, GLOBALFOUNDRIES Inc. (United States)

Jeong-Ho Yeo, SAMSUNG Electronics Company, Ltd. (Korea, Republic of)

Masaki Yoshioka, XTREME technologies GmbH (Germany)

\section{Session Chairs}

1 Invited I

Jos P. Benschop, ASML Netherlands B.V. (Netherlands)

Shinji Okazaki, Gigaphoton Inc. (Japan)

2 EUV Resists: Joint Session with Conferences 8679 and 8682

Robert L. Brainard, University at Albany (United States)

George G. Barclay, Dow Advanced Materials (United States)

3 Sources

Li-Jui Chen, Taiwan Semiconductor Manufacturing Company Ltd. (Taiwan)

Michael J. Lercel, SEMATECH Inc. (United States)

4 Mask I

Frank Goodwin, SEMATECH Inc. (United States)

Naoya Hayashi, Dai Nippon Printing Company, Ltd. (Japan)

5 Resist Outgassing

Soichi Inove, EUVL Infrastructure Development Center, Inc. (Japan)

Kurt G. Ronse, IMEC (Belgium)

6 Optics and Metrology

Katsuhiko Murakami, Nikon Corporation (Japan)

Winfried Kaiser, Carl Zeiss SMT GmbH (Germany)

$7 \quad$ OPC and Modeling

Gregory R. McIntyre, IBM Corporation (United States)

Eric M. Panning, Intel Corporation (United States)

8 EUV Resists

Chris S. Ngai, Applied Materials, Inc. (United States)

Thomas I. Wallow, GLOBALFOUNDRIES Inc. (United States) 
9 High NA and Magnification

Ted Liang, Intel Corporation (United States)

Jan Hendrik Peters, Carl Zeiss SMS GmbH (Germany)

10 Mask II

Stanley E. Stokowski, KLA-Tencor Corporation (United States)

Tsutomu Shoki, HOYA Corporation (Japan)

11 Invited II

Daniel A. Corliss, IBM Corporation (United States)

Seong-Sue Kim, SAMSUNG Electronics Company, Ltd. (Korea, Republic of)

Poster Session

Patrick P. Naulleau, Lawrence Berkeley National Laboratory (United States)

Obert R. Wood II, GLOBALFOUNDRIES Inc. (United States) 
Proc. of SPIE Vol. $8679867901-18$

Downloaded From: https://www.spiedigitallibrary.org/conference-proceedings-of-spie on 26 Apr 2023 Terms of Use: https://www.spiedigitallibrary.org/terms-of-use 